\title{
From Spreadsheets to Simulation-Based Aircraft Conceptual Design
}

\author{
Mehdi Ghoreyshi ${ }^{1}$ \& Russell M. Cummings ${ }^{1}$ \\ ${ }^{1}$ High Performance Computing Research Center, U. S. Air Force Academy, Colorado, United States \\ Correspondence: Mehdi Ghoreyshi, High Performance Computing Research Center, U. S. Air Force Academy, \\ Colorado 80840, United States. E-mail: Mehdi.Ghoreyshi@usafa.edu
}

Received: March 8, $2014 \quad$ Accepted: March 31, $2014 \quad$ Online Published: April 21, 2014
doi:10.5539/apr.v6n3p64 $\quad$ URL: http://dx.doi.org/10.5539/apr.v6n3p64

\begin{abstract}
An automated, simulation-based aircraft design process allows for the prediction of unanticipated problems early in the design stage, leading to reduced turn-around time and development cost. Having reliable, and affordable (fast) design tools is crucial to achieving this level of automation in design process. An example of this is illustrated for a jet trainer aircraft using two aircraft design codes: Jet Designer and CEASIOM. A set of aerodynamic methods with different degrees of fidelity and computational expense is considered, with the limitations of each method provided. In particular, this paper examines the challenges that CFD-based aircraft design poses to a designer, including: a) the cost of generation of large data tables, b) the automated handling of geometry, c) treating control surface deflections, d) and calculation of dynamic derivatives using CFD. A Kriging-based sampling approach was used for generating aerodynamic tables with a reasonable computational cost compared with a brute-force approach. For Euler calculations, an automated CAD and mesh generation approach from a geometry description was used. It is demonstrated that application of Euler solutions to low fidelity aircraft geometry shows the expected design trends. Also, results show that the wave drag at transonic speeds can be predicted with Euler equations, but not with vortex lattice or Digital DATCOM. The treatment of control surface deflections was also investigated for the vortex lattice solver and the Euler code. Transpiration boundary condition approach was used in the Euler code to model the flap surface movements, although this approach is limited to small control surface deflections. The calculated aero tables form each aero source were used next to study the vehicle flying qualities. Results presented demonstrate the validity and feasibility of the simulation-based approach for aircraft conceptual design.
\end{abstract}

Keywords: Kriging, overset grid, transpiration method, dynamic derivatives, flight handling qualities

\section{Nomenclature}

$a$

$b$

$c$

$C_{D}$

$C_{L}$

$C_{L \alpha}$

$C_{L q}$

$C_{m}$

$C_{m \alpha}$

$C_{m q}$

$C_{m \delta e}$

$C_{Y}$

$C_{Y \beta}$

$k$

D acoustic speed, $\mathrm{m} / \mathrm{s}$

wing span, $m$

wing chord, $\mathrm{m}$

drag coefficient, $D / q_{\infty} S$

lift coefficient, $L / q_{\infty} S$

lift curve slope, $1 / \mathrm{rad}$

lift derivative with respect to normalized pitch rate, $1 / \mathrm{rad}$

pitching moment coefficient, $m / q_{\infty} S c$

pitching moment slope, $1 / \mathrm{rad}$

pitching moment derivative with respect to normalized pitch rate, $1 / \mathrm{rad}$

pitching moment derivative with respect to elevator deflection, 1/rad

side-force coefficient, $Y / q_{\infty} S$

side-force derivative with respect to side-slip angle, $1 / \mathrm{rad}$

reduced frequency, $\omega c / 2 \mathrm{~V}$

drag force, $\mathrm{N}$ 


$\begin{array}{ll}L & \text { lift force, } \mathrm{N} \\ L_{f u s} & \text { Total fuselage length, } \mathrm{m} \\ M & \text { Mach number, } \mathrm{V} / \mathrm{a} \\ M A C & \text { mean aerodynamic chord, } \mathrm{m} \\ m & \text { pitching moment, } \mathrm{N}-\mathrm{m} \\ p & \text { pressure, } \mathrm{Pa} \\ \dot{q} & \text { time rate of pitch rate, } \mathrm{rad} / \mathrm{s}^{2} \\ q & \text { pitch rate, } \mathrm{rad} / \mathrm{s} \\ q_{\infty} & \text { dynamic pressure, } \mathrm{Pa} \\ R e & \text { Reynolds number, } \rho V c / \mu \\ S & \text { reference area, } \mathrm{m}^{2} \\ T & \text { oscillation period, } \mathrm{s} \\ T A S & \text { true air speed, } \mathrm{m} / \mathrm{s} \\ t & \text { time, } \mathrm{s} \\ V & \text { velocity, } \mathrm{m} / \mathrm{s} \\ X_{h} & \text { Horizontal tail apex distance from nose, } \mathrm{m} \\ Y & \text { side force, } \mathrm{N}\end{array}$

\section{Greek}

$\dot{\alpha}$

$\alpha$

$\alpha_{A}$

$\beta$

$\Lambda$

$\Gamma$

$\alpha_{0}$

$\omega$

$\rho$

$\mu$ normalized time rate of angle of attack, 1/rad

angle of attack, rad

pitch amplitude, rad

side-slip angle, rad

wing sweep angle, deg

wing dihedral angle, deg

mean angle of attack, rad

circular frequency, $\mathrm{rad} / \mathrm{s}$

density, $\mathrm{kg} / \mathrm{m}^{3}$

air viscosity

\section{Introduction}

The aircraft design process consists of three main phases: a) conceptual, b) preliminary, and c) detailed design (Brandt et al., 2004). Traditionally, conceptual design starts by assuming basic mission requirements of range, payload, cruise speed, altitude, and take-off distance (Ferreri, 2003). The general size and configuration will be described using conventional engineering drawing and spreadsheet data to meet mission requirements (Fielding, 1999). From this geometry description, spreadsheets provide an estimation of aircraft aerodynamics and weights by using various methods, typically based on comparisons to previous designs (Raymer, 2006). Next, rough estimations of drag and lift forces are used to find the payload to range ratio, stability, and performance data. To validate the concept design, computer simulations and physical models of the design are built and tested in the preliminary design phase (Brandt et al., 2004). Computational Fluid Dynamics (CFD) simulations are likely used in this phase to simulate flow around aircraft for qualitative studies and diagnostics purposes rather than to generate a new aerodynamic model for stability and control predictions (Kundu, 2010). As designs move forward into a detailed design phase, a more rigorous analysis of aerodynamics, dynamics, structure, and system architecture begins (Austin, 2010). A prototype is often built and tested at the end of the aircraft design cycle. To remedy any detected problem(s) in prototype testing, sometimes the whole design cycle needs to be repeated. This turn-around time will increase development cost by thousands of millions of dollars, therefore it is essential to reduce any risk during prototype testing by early detection of aerodynamic-related problems (Huenecke, 1987). 
For fighter configurations, aerodynamic challenges in conceptual design include prediction of vortical flow and shock waves which can lead to uncommanded motion and uncontrollable departure in flight testing. Some recent examples include aircraft such as F/A-18, F-18E, and F-22 (Chambers \& Hall, 2004; Hall et al., 2005; Bowers et al., 1996; Wang \& Iliff, 2004; Moses, 2005; Potoczsky \& Moses, 2005). The development of a reliable computational tool for prediction of these important issues would allow the designers to screen different configurations prior to building the first prototype, reducing overall cost and limiting risks. This provides motivation to move towards CFD simulations based on the state-of-the-art computer-aided concept, since this approach, in principle, has few limitations related to geometry. At the highest practical level, simulation based on the Reynolds Averaged NavierStokes (RANS) equations have the potential to predict the full range of regimes of interest to the designer. A number of problems need to be addressed for the routine use of CFD for conceptual design: a) the cost of generation of large data tables (Ghoreyshi et al., 2009), b) the automated handling of geometry (Berard et al., 2008), c) treating control surface deflections (Da Ronch et al., 2011), d) and calculation of dynamic derivatives (Da Ronch et al., 2012). More details are given below.

There are a number of CFD-based aerodynamic models described in the literature. A traditional stability and control derivative model can be obtained by differentiating CFD forces and moments with respect to angle of attack and side-slip (Park et al., 1999). This model is only valid in the linear regimes of the flight envelope and hence the nonlinear effects from vortical flows and shock waves cannot be reconciled. To overcome this deficit, researchers at NASA Ames suggested a brute-force approach to populate a large database of aerodynamic coefficients for the full flight envelope (Murman et al., 2002; Rogers et al., 2003). They found that a reasonable database size for stability and control study would be on the order of 30,000 data points (Murman et al., 2002), which makes the approach unrealistic for RANS calculations since a separate run is required for each data point (Jameson, 1999).

Efforts have been made to recover these large tables using surrogate-based algorithms (Ghoreyshi et al., 2009; Mackman et al., 2013). An approach to generate aerodata with an acceptable cost was proposed by Ghoreyshi et al. (2009). Two scenarios were considered, based on: (1) a requirement for tables for a completely new design and (2) updating tables for an existing design which is being altered. It was shown that tables which are practically useful could be generated using on the order of 1000 calculations under the first scenario and 100 calculations under the second scenario. An issue of tabular database modeling is related to the unsteady aerodynamics: the aero tables lack the time hysteresis arising from unsteady vortical flows (Ghoreyshi et al., 2011). Thus, a number of Reduced Order Models (ROM) were developed for modeling unsteady aerodynamics at such conditions. Once a ROM has been created, it can be used for rapid prediction of unsteady aerodynamic loads from motion descriptions (Ghoreyshi et al., 2012). Our focus in this paper is quasi-steady aerodynamics and hence tabular data are used. The methods of generating these tables using a Surrogate modeling are detailed.

The second issue for creating CFD-based aerodynamic models is related to the geometry modeling: the use of CFD simulations in conceptual design needs an automated solid modeling capability with a rapid mesh generator. A problem of using a conventional CAD system in conceptual design is that generated spline surfaces do not correspond to the parameters that a designer uses to describe the conceptual aircraft geometry (such as wing sweep or thickness) (Rodriguez \& Sturdza, 2006). A new CAD capability that allows geometry modeling from a small number of design parameters is needed. Some recent efforts are Boeing's proprietary tool, General Geometry Generator (Bowcutt, 2003), NASA's Rapid Aircraft Modeler (McCormick, 2002; Gloudemans et al., 1996), KTH (Royal Institute of Technology)'s CADac (Berard et al., 2008), Stanford's AEROSURF (Alonso et al., 2003) and KTH's Surface Modeler (SUMO) (Tomac \& Eller, 2010). Boeing's GGG software, written in the Python language, has a library of lofting codes to create a parameterized geometry model for conceptual aircraft design. NASA's RAM generates a geometry model and a surface mesh from aircraft parameters such as wing aspect ratio, taper ratio, span, and angles of twist, sweep, and dihedral. The CADac and AEROSURF software are CAPRI-based applications that link the CAD package and the aircraft design software that requests the variation in the geometry. CAPRI (Computational Analysis Programming Interface) (Haimes, 1998) offers a coupling to any supported CAD package by using API to access the geometry and topological data. SUMO is written in $\mathrm{C}^{++}$and has a library of geometric primitives based on B-spline curves and surfaces to create a parameterized watertight surface model of a concept aircraft. Since the number of design parameters to describe concept designs are small, these solid models would likely have rough joint and junction geometries that would prompt flow separation prediction by a RANS simulation. In this sense high fidelity simulations on low fidelity geometries may provide misleading information about the underlying properties of the design. This paper examines the rough geometry effects by comparing design prediction trends with available data. 
The next issue occurs when dealing with simulation of control surfaces: changing a control-surface deflection often means regeneration of the grid. This can be a very time-consuming and labor intensive task as the number of moving surfaces increases. A number of methods for treating control surfaces are available that eliminate the need to regenerate grids. Vallespin et al. (2011), for example, used mode shapes for the control surface deflections. Each mode shape specifies the displacement of the grid points on the aircraft surface for a particular control surface. However, this approach is limited for large deflection angles. Another method is a transpiration boundary condition that imposes a nonzero transpiration velocity on the surface of an undeflected control surface. This velocity component is normal to the actual deflected surface and thus all calculations can be run virtually on the clean configuration grid. This technique imposes a limitation on the amount of maximum and minimum deflections (Da Ronch et al., 2011). Also, overset unstructured grid techniques, with a number of overlapping grids are found useful for modeling control surface deflection (Nakahashi, 2000). Independent meshes are generated around different body elements and then the discretized flow equations are solved by using an interpolation technique. This paper investigates the use of transpiration boundary conditions for control surface deflections because of its relative simplicity.

The last issue is related to the calculation of dynamic derivatives: a method is required to extract and separate dynamic derivatives from time-accurate CFD solutions. Pitch, yaw, and roll oscillations are often used to extract dynamic effects in terms of derivatives. The time-histories of aerodynamic coefficients undergoing these motions depends on the motion amplitude, mean angle, reduced frequency, and in particular the selected time-step and the flow solver used. Also, the aerodynamic responses consist of in-phase and out-phase terms. The mathematical modeling of dynamic terms from CFD calculations are therefore discussed.

In this paper a simulation-based design study of a jet trainer is considered. The paper begins with a description of the geometry handling and the prediction tools. The test case, geometry definition, and mesh generation are then detailed. Next, the geometry impacts on aerodynamic predictions are detailed including the effects of inlet modeling in drag force predictions. A validation of the aerodynamic tools is made against wind tunnel and flight test data. Validation cases associated with small and large flap deflection angles, and low and high speeds are considered. Finally, aero tables are generated and used to study the vehicle flying qualities.

\section{Formulation}

\subsection{Geometry}

For a computer-aided analysis and optimization, the geometry of the initial concept must be described. The Computerized Environment for Aircraft Synthesis and Integrated Optimization Methods (CEASIOM) (von Kaenel et al., 2008), the design code used in the current paper, incorporates a parameterized description of the geometry given by the Jet Designer code. Jet Designer is a low fidelity code developed at the United States Air Force Academy (USAFA) for the design of jet aircraft. The geometry parameters include lifting surfaces and a fuselage; the fuselage is defined using 20 cross sections. The lifting surfaces are defined using the apex position and placement, leading edge sweep angle, dihedral angle, span, wing area, taper ratio. The vertical tail can have a lateral displacement with a tilt angle. The strake is defined by span and leading edge sweep angle. A number of aircraft can be defined using this definition, however, aerodynamic prediction tools in the code are limited to straight wing and conventional configurations.

CEASIOM starts from the Jet Designer geometry and allows the design to be refined by adding winglet and cranked wings. In general, an aircraft geometry can be defined in CEASIOM with approximately 100 design variables. The geometry description is then interpreted for use in Digital DATCOM and the vortex lattice solver of Tornado. Of particular interest is the approach used to perform automatic mesh generation for the Euler solver. The surface modeling package, SUMO, produces a surface model, and its triangulation. The model can be passed to an extended CAD system or mesh generator as a standard CAD interface file, and the surface mesh is directed to a tetrahedral volume mesh generator.

SUMO is a rapid geometry modeling tool for parametrically-defined aircraft configurations. The code, written in $\mathrm{C}^{++}$, has a library of geometric primitives based on B-spline curves and surfaces to create a parameterized watertight surface model of the complete Jet aircraft. The way SUMO defines a control surface is to input the inboard and outboard span locations as well as chord lengths, while an additional midboard section is needed for a cranked wing.

The automatic mesh generation tool in SUMO provides an unstructured surface mesh. The mesh control parameters are estimated from the model geometrical features, such as radii of curvature and the presence of sharp edges. From 
the surface mesh, unstructured volume meshes can be automatically generated using the tetrahedral mesh generator TetGen (http://tetgen.berlios.de/). This computational grid is then used to solve the Euler equations of an external flow past the aircraft, assuming an inviscid solid wall boundary condition for the engine inlet and exhaust.

\subsection{Aerodynamics}

\subsubsection{Jet Designer}

The Jet Designer program (Marshall et al., 2005) provides a derivative-based aerodynamics model for fighter configurations. The static, control, and dynamic derivatives in longitudinal and lateral directions are estimated from methods in Roskam (2000) and Yechout et al. (2003) for a given geometry definition and flight conditions. The aerodynamic model is adequate for low angles of attack or in the linear and steady flight dynamic regimes. The software has an iterative scheme that allows aircraft shape optimization. The primary measures of merit for the optimization are sized takeoff gross weight and sized mission fuel burn.

\subsubsection{Digital DATCOM}

The U.S.A.F. Stability and Control Digital DATCOM is a semi-empirical approach which rapidly produces the aerodynamic derivatives based on geometry details and flight conditions. DATCOM was primarily developed to estimate aerodynamic derivatives of conventional configurations (Razgonyaev \& Mason, 1995; Williams \& Vukelich, 1979), and hence the approach is not likely to be accurate (or possible) for non-conventional designs. Other DATCOM limitations are discussed by Blake (1985). Digital DATCOM has been implemented into CEASIOM to run directly from the Jet aircraft geometric data.

\subsubsection{Vortex Lattice Solver}

The vortex lattice solver of TORNADO (Melin, 2000) can predict a wide range of aircraft stability and control aerodynamic derivatives using a vortex lattice approach. The code models various lifting surfaces such as wing, fin, and canard. The fuselage is modeled by a thin plate. For control surface deflections, the vortex points located at the trailing edge of the flap are rotated around the hinge line which makes the wake change direction slightly (Melin, 2000).

\subsubsection{EDGE Solver}

Edge (Eliasson, 2002) is a parallelized CFD package developed by FOI (the Swedish Defence Research Agency). The code can be applied to 2D/3D viscous (RANS) or inviscid (Euler) compressible flow problems on unstructured grids with arbitrary elements and is used in Euler mode in CEASIOM. Also, Edge allows for both steady state and time-accurate calculations. The space discretisation exploits a node-centered finite-volume technique using an edge-based data structure. The computational elements are a set of non-overlapping cells formed as the dual of the primary tetrahedral mesh. Explicit Runge-Kutta time stepping integrates the discrete equations in time. Accelerated convergence to steady state is promoted using agglomeration multigrid and implicit residual smoothing. A Matlab interface was written to automate the preprocessing and run the CFD calculations for static, dynamic, and control coefficients. The interface has postprocessing utilities for extraction of the integrated aerodynamic coefficients, either in the body or wind axes, from the surface solution. The way EDGE calculates the aerodynamics of control surface deflections is based on the use of transpiration boundary conditions. In this approach, instead of moving the grid, the wall velocity component normal to the actual deflected surface is prescribed. Such an approach eliminates the need for mesh deformation, thus all calculations can be run virtually on the clean configuration grid. On the other hand, this imposes a limitation on the amount of maximum and minimum deflections which are physically feasible.

\subsubsection{Cobalt Solver}

The commercially available code, Cobalt (Strang et al., 1999) solves the unsteady, three-dimensional and compressible Navier-Stokes equations. The Navier-Stokes equations are discretised on arbitrary grid topologies using a cell-centered finite volume method. Second-order accuracy in space is achieved using the exact Riemann solver of Gottlieb and Groth (Gottlieb \& Groth, 1988), and least squares gradient calculation using QR factorization. In order to accelerate the discretized system, a point-implicit method using analytic first-order inviscid and viscous Jacobian is used. A Newtonian sub-iteration method is used to improve time accuracy of the point-implicit method. The resulting method is second-order accurate in time. Tomaro et al. (1997) converted the code from explicit to implicit, enabling CFL numbers as high as $10^{6}$. The Cobalt solver has been used at the Air Force Seek Eagle Office (AFSEO) and the United States Air Force Academy (USAFA) for a variety of unsteady nonlinear aerodynamic problems of maneuvering aircraft (Forsythe et al., 2002; Forsythe \& Woodson, 2005; Morton et al., 2002; Forsythe 
et al., 2004; Jeans et al., 2009).

Cobalt is based on an arbitrary Lagrangian-Eulerian formulation and hence allows all translational and rotational degrees of freedom (Jirásek \& Cummings, 2010). For the control surface simulations, the use of overset grid is available. The code can simulate both free and specified six degree of freedom (6DoF) motions. The rigid motion is specified from a motion input file. For the rigid motion the location of a reference point on the aircraft is specified at each time step. In addition the rotation of the aircraft about this reference point is also defined using the rotation angles of yaw, pitch, and bank.

\subsection{Surrogate-Based Generation of Aerodynamic Tables}

A nonlinear quasi-steady aerodynamic model is considered for this work. For longitudinal coefficients the model is written as:

$$
C_{i}=C_{i 0}(\alpha, M, \beta)+\bar{C}_{i q}(\alpha, M, q) \cdot \frac{c q}{2 V}+C_{i \delta}(\alpha, M, \delta) \cdot \delta
$$

where $i=L, D$, and, $m$, representing lift, drag, and pitching moment coefficients, respectively. $\delta$ includes longitudinal and lateral control deflections, and $q$ is the pitch rate. $\bar{C}_{i q}$ is named the out-of-phase pitch derivative which includes the effects of $q$, and $\dot{\alpha}$. For lateral coefficients the model is:

$$
C_{i}=C_{i 0}(\alpha, M, \beta)+\bar{C}_{i p}(\alpha, M, p) \cdot \frac{b p}{2 V}+\bar{C}_{i r}(\alpha, M, r) \cdot \frac{b r}{2 V}+C_{i \delta}(\alpha, M, \delta) . \delta
$$

where $i=Y, l$, and, $n$ which denote side force, rolling, and yawing moment coefficients, respectively. The terms $p$, and $r$ are the roll and yaw rates. The state and control dependencies given in Equations (1)-(2) can be found from tabulated data, and then an interpolation model is used to estimate aerodynamic coefficients. For an aircraft with conventional control surfaces of elevator, rudder, and aileron the format of the aerodynamic tables is shown in Table 1.

\begin{tabular}{|c|c|c|c|c|c|c|c|c|c|c|c|c|c|c|}
\hline$\alpha$ & $M$ & $\beta$ & $\delta_{\text {ele }}$ & $\delta_{\text {rud }}$ & $\delta_{\text {ail }}$ & $q$ & $p$ & $r$ & $C_{L}$ & $C_{D}$ & $C_{m}$ & $C_{Y}$ & $C_{l}$ & $C_{n}$ \\
\hline $\mathrm{X}$ & $\mathrm{X}$ & $\mathrm{X}$ & - & - & - & - & - & - & - & $\mathrm{X}$ & $\mathrm{X}$ & $\mathrm{X}$ & $\mathrm{X}$ & $\mathrm{X}$ \\
\hline $\mathrm{X}$ & $\mathrm{X}$ & - & $\mathrm{X}$ & - & - & - & - & - & - & $\mathrm{X}$ & $\mathrm{x}$ & $\mathrm{x}$ & $\mathrm{X}$ & $\mathrm{X}$ \\
\hline$X$ & $\mathrm{X}$ & - & - & $\mathrm{X}$ & - & - & - & - & - & $\mathrm{X}$ & $\mathrm{x}$ & $\mathrm{x}$ & $\mathrm{X}$ & $\mathrm{X}$ \\
\hline $\mathrm{X}$ & $\mathrm{X}$ & - & - & - & $\mathrm{X}$ & - & - & - & - & $\mathrm{x}$ & $\mathrm{X}$ & $\mathrm{x}$ & $\mathrm{X}$ & $\mathrm{X}$ \\
\hline $\mathrm{X}$ & $\mathrm{X}$ & - & - & - & - & $\mathrm{X}$ & - & - & - & $\mathrm{X}$ & $\mathrm{x}$ & $\mathrm{x}$ & $\mathrm{X}$ & $\mathrm{X}$ \\
\hline $\mathrm{X}$ & $\mathrm{X}$ & - & - & - & - & - & $\mathrm{X}$ & - & - & $\mathrm{X}$ & $\mathrm{x}$ & $\mathrm{X}$ & $\mathrm{x}$ & $\mathrm{X}$ \\
\hline $\mathrm{X}$ & $\mathrm{X}$ & - & - & - & - & - & - & $\mathrm{X}$ & - & $\mathrm{X}$ & $\mathrm{x}$ & $\mathrm{X}$ & $\mathrm{X}$ & $\mathrm{X}$ \\
\hline $\mathrm{X}$ & $\mathrm{X}$ & - & - & - & - & - & - & - & $\mathrm{x}$ & $\mathrm{X}$ & $\mathrm{x}$ & $\mathrm{X}$ & $\mathrm{x}$ & $\mathrm{X}$ \\
\hline
\end{tabular}

Table 1. Aerodynamic database format, "x" indicates a column vector of non-zero elements

This large table consists of eight sub-tables, each having three aerodynamic variables, where two variables are the angle of attack, $\alpha$, and Mach number, $M$. These tables are relatively large and hence would be computationally expensive to generate using a brute-force approach. In this paper, a Kriging predictor (Jones et al., 1998) is used to approximate aerodynamic loads in each sub-table using a number of observed values (samples). Assume that $n$ samples are available for a function with $k$ independent variables. Each sample is denoted as $x^{(i)}=\left(x_{1}^{(i)}, \ldots, x_{k}^{(i)}\right)$ with corresponding observations (responses) $y^{(i)}=y\left(x^{(i)}\right)$, for $i=1, \ldots, n$. A Kriging function is used to approximate the target function at a new point of $x^{*}$ as

$$
\hat{y}\left(x^{*}\right)=\mu+\epsilon
$$

where $\mu$ is the mean value and $\epsilon$ is the normally distributed error term with zero mean and variance $\sigma^{2}$. To compute the Kriging model, the model parameters are quantified using the Maximum Likelihood Estimator (MLE), as described by Jones et al. (1998).

In general, we need a systematic method for the generation of samples for Kriging that ensures the uncertainty in the prediction of the target function is minimized. In order to minimize this uncertainty, we exploit the sample generation based on Kriging predicted Mean Squared Error (MSE). The Kriging model provides an estimation 
of the MSE, which is zero at observed points and increases as the distance between samples increases, and can be used as the criteria for sample generation. In this paper, a sample is generated at each iteration at the location where the MSE is maximum. The sampling stops if the maximum number of iterations is reached or MSE becomes smaller than some reasonable tolerance.

\subsection{Calculation of Dynamic Derivatives Using CFD}

The estimation of dynamic derivatives is obtained by imposing a forced sinusoidal motion around the aircraft center of gravity which mimics how derivatives are obtained in wind tunnel testing. For the computation of the longitudinal dynamic derivative values from the time-histories of the forces and moments, it is assumed that the aerodynamic coefficients are linear functions of angle of attack, $\alpha$, pitching angular velocity, $q$, and rates $\dot{\alpha}$ and $\dot{q}$. To illustrate, the increment in the longitudinal aerodynamic coefficients (lift, drag and pitching moment) with respect to the mean value during the applied pitching sinusoidal motion is formulated as:

$$
\begin{gathered}
\Delta C_{j}=C_{j_{\alpha}} \Delta \alpha+C_{j_{\dot{\alpha}}} \frac{c}{2 U_{\infty}} \dot{\alpha}+C_{j_{q}} \frac{c}{2 U_{\infty}} q+C_{j_{\dot{q}}}\left(\frac{c}{2 U_{\infty}}\right)^{2} \dot{q} \\
\text { for } j=L, D, \text { and } m
\end{gathered}
$$

The harmonic motion in pitch defines the kinematic relations for the angle of attack, pitching angular velocity, and rate as:

$$
\begin{aligned}
& \Delta \alpha=\alpha_{A} \sin (\omega t) \\
& \dot{\alpha}=q=\omega \alpha_{A} \cos (\omega t) \\
& \ddot{\alpha}=\dot{q}=-\omega^{2} \alpha_{A} \sin (\omega t)
\end{aligned}
$$

Equation (4) can then be rewritten as:

$$
\Delta C_{j}=\alpha_{A} \bar{C}_{j_{\alpha}} \sin (\omega t)+\alpha_{A} k \bar{C}_{j_{q}} \cos (\omega t)
$$

where $k=\omega c /\left(2 U_{\infty}\right)$ indicates the non-dimensional reduced frequency of the applied motion. The in-phase and out-of-phase components of $\Delta C_{j}$, respectively indicated as $\bar{C}_{j_{\alpha}}$ and $\bar{C}_{j_{q}}$ (Klein et al., 1997), are defined as

$$
\begin{aligned}
& \bar{C}_{j_{\alpha}}=\left(C_{j_{\alpha}}-k^{2} C_{j_{\dot{q}}}\right) \\
& \bar{C}_{j_{q}}=\left(C_{j_{\dot{\alpha}}}+C_{j_{q}}\right)
\end{aligned}
$$

The dynamic derivative values can be calculated taking the first Fourier coefficients of the time history of $\Delta C_{j}$ over $n_{c}$ cycles

$$
\begin{aligned}
& \bar{C}_{j_{\alpha}}=\frac{2}{\alpha_{A} n_{c} T} \int_{0}^{n_{c} T} \Delta C_{j}(t) \sin (\omega t) d t \\
& \bar{C}_{j_{q}}=\frac{2}{k \alpha_{A} n_{c} T} \int_{0}^{n_{c} T} \Delta C_{j}(t) \cos (\omega t) d t
\end{aligned}
$$

where $T=2 \pi / \omega$ is the period of one cycle of unsteadiness. The model formulation given in Equation (4) is adequate for aircraft operating at low angles of attack or in linear and steady aerodynamic flight regimes, with the out-of-phase giving a good approximating of the aerodynamic damping.

\section{Test Case}

The Northrop T-38 Talon (as shown in Figure 1) is a two-seat advanced jet trainer powered by two J85-GE-5 turbojet engines (Brandt et al., 2004). The aircraft has a ceiling of 50,000 feet, a climb rate of 34,000 feet per minute, and can fly at speeds as high as Mach 1.2, so it became the world's first supersonic trainer. The vehicle is a variant of the F-5A tactical fighter, (Mueller, 1999) with a conventional design featuring a swept wing with an area of $S=170 \mathrm{ft}^{2}$, span of $25.25 \mathrm{feet}$, aspect ratio of 3.75, taper ratio of 0.2 , and NACA $65 \mathrm{~A}-004$ airfoil section. 

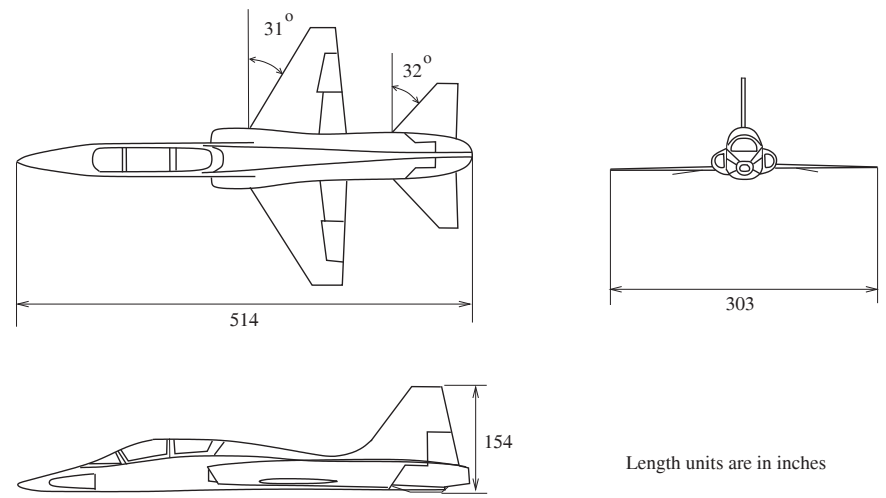

Length units are in inches

Figure 1. The T-38 Talon aircraft

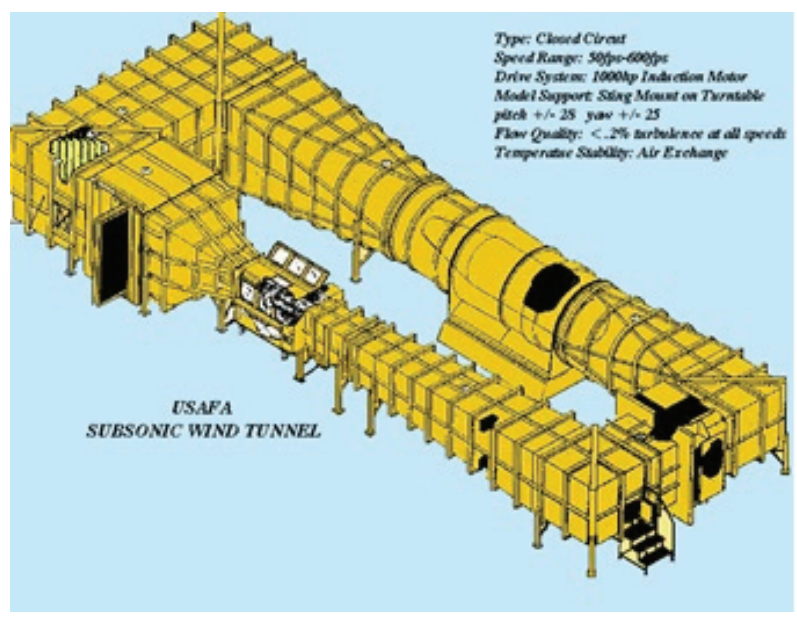

Figure 2. The USAFA subsonic wind tunnel schematic

The static experiments for the T-38 were conducted in the Subsonic Wind Tunnel (SWT) facility at USAFA. This closed-loop tunnel as shown in Figure 2 has an $8 \mathrm{ft}$ long test section with a test section cross-section $3 \mathrm{ft}$ by $3 \mathrm{ft}$. The tunnel can achieve speeds in excess of Mach 0.5. The flow conditions in the experiments are $M=0.2975$, $R e=6.4 \times 10^{5}$, and $\beta=0$. The T-38 wind tunnel model has a wing span of $16.5 \mathrm{in}$, and a mean aerodynamic chord of $5.05 \mathrm{in}$. Also, flight test data at 30,000 ft are available from Brandt el al. (2004) for clean and flapped T-38 configurations. The data correspond to $M=0.4$ and flap angles of $10^{\circ}$, and $45^{\circ}$.

The T-38 geometry described in Jet Designer is converted to models for use in Digital DATCOM, Tornado, and EDGE. Figure 3(a) shows the T-38 model and mesh in EDGE. The mesh was generated automatically using Tetgen. The grid corresponds to a full-aircraft model without engine inlet, and with the far-field modeled by a large sphere with a radius of $234 \mathrm{ft}$. The grid has approximately 387,000 nodes and 2.5 million edges. Also, two RANS meshes were generated from the half geometry model of the T-38 with and without an engine air inlet. The meshes were generated in two steps. In the first step, the inviscid tetrahedral mesh was generated using the ICEM-CFD code. This mesh was then used as a background mesh by TRITET (Tyssel, 2000) which builds prism layers using an advancing front technique. TRITET rebuilds the inviscid mesh while respecting the size of the original inviscid mesh from ICEM-CFD. The generated RANS grids are shown in Figures 3(b)-(c). The RANS grids have approximately $6 \mathrm{M}$ points and $12 \mathrm{M}$ cells. All RANS simulations were run on the Cray XE6 machine at the Engineering Research Development Center (ERDC); machine name is Garnet with $2.7 \mathrm{GHz}$ core speed. 


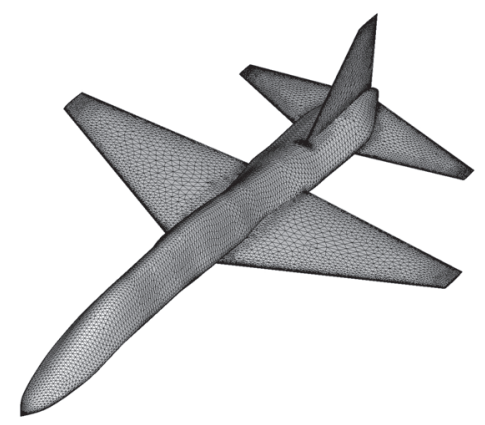

(a) Euler mesh

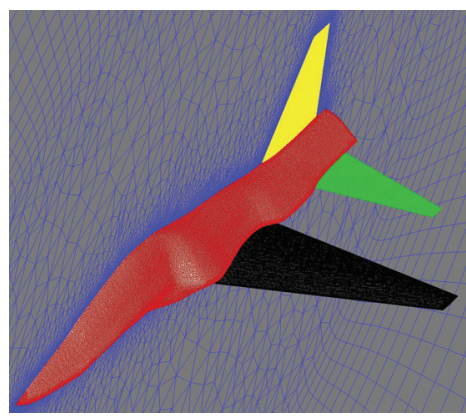

(b) RANS mesh of blocked inlet model

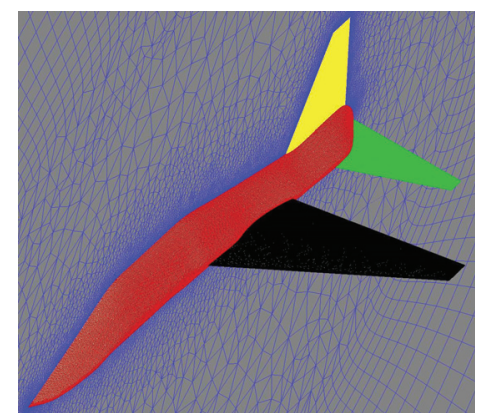

(c) RANS mesh of no inlet model

Figure 3. Euler and RANS mesh overview

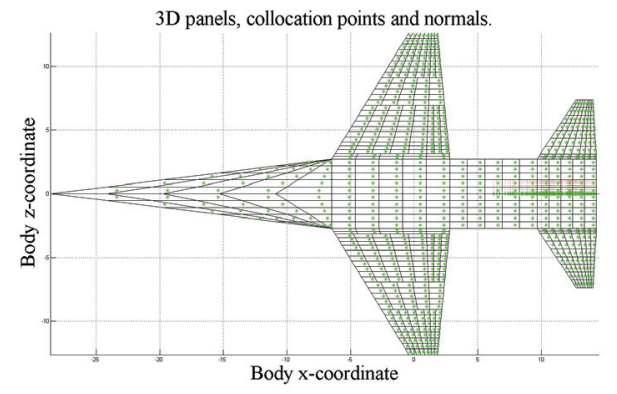

(a) FWVH- panels including fuselage

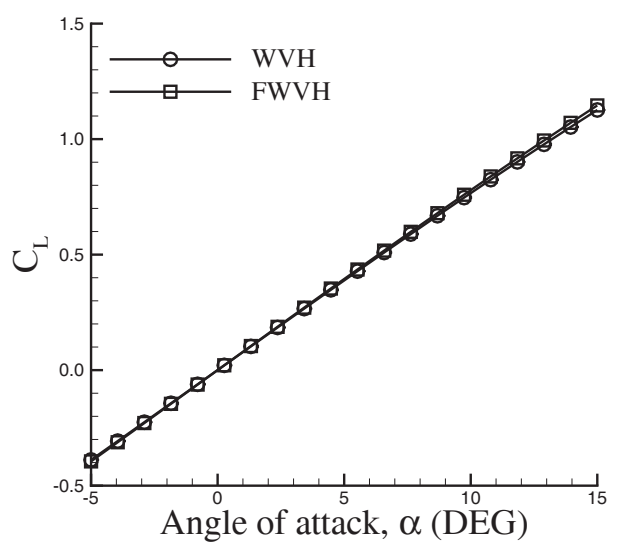

(c) Lift coefficient

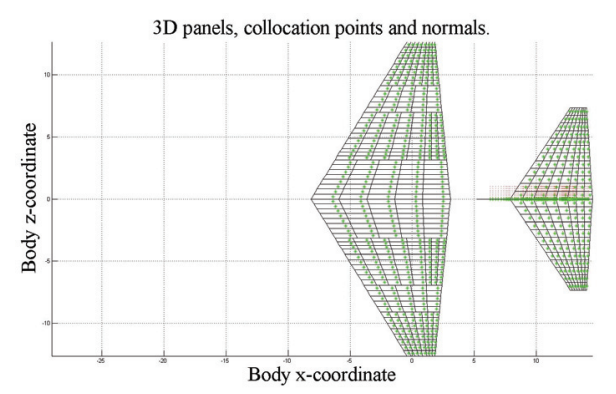

(b) WVH- panels without fuselage

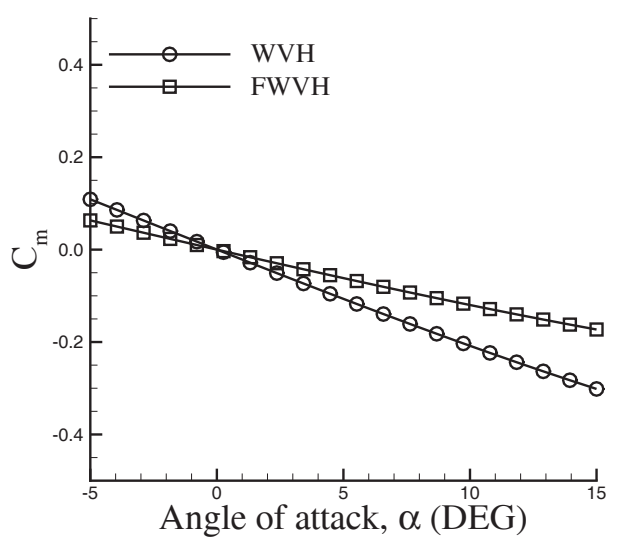

(d) Pitching moment coefficient

Figure 4. The effects of fuselage on Tornado lift and pitching moment predictions. The Mach number is $M_{\infty}=0.4$ 


\section{Results}

Tornado panels for the T-38 are shown in Figures 4(a)-(b) representing the wing, vertical and horizontal tail (WVH) model and a simplified fuselage model with lifting surfaces (FWVH). The fuselage was modeled as a thin flat plate. The total number of panels are 768 and 860 for WVH and FWVH, respectively. The effects of fuselage modeling on the lift and pitching moment predictions are shown in Figures 4(c)-(d). As might be expected, the fuselage has no appreciable effect on the produced lift force, but it shifts the aerodynamic center rearwards and consequently changes the pitching moment slope. Tornado prediction results in subsequent sections refer to the FWVH model unless otherwise stated.

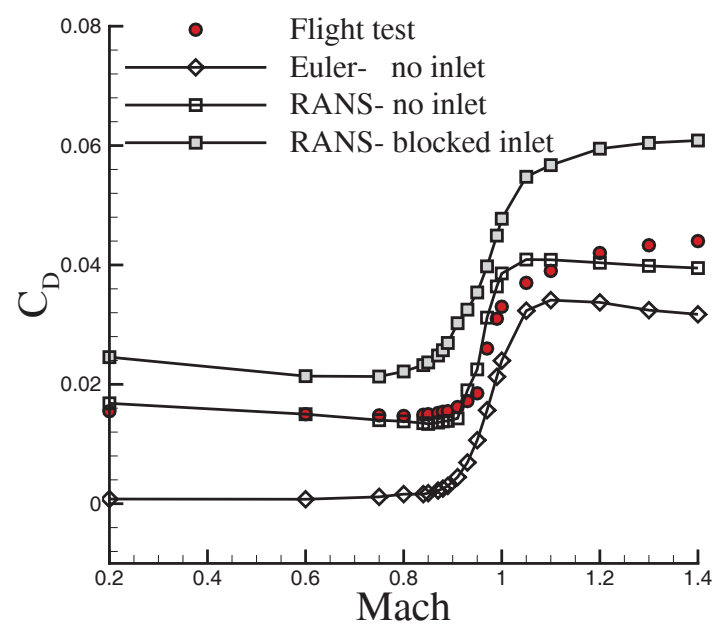

(a) Drag coefficient

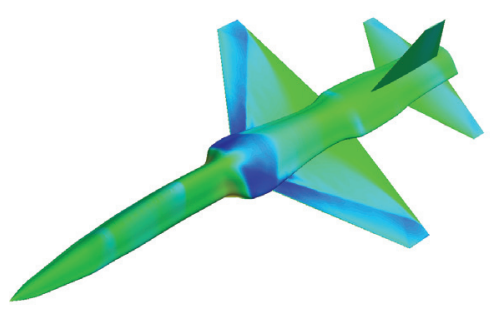

(b) RANS - Blocked inlet

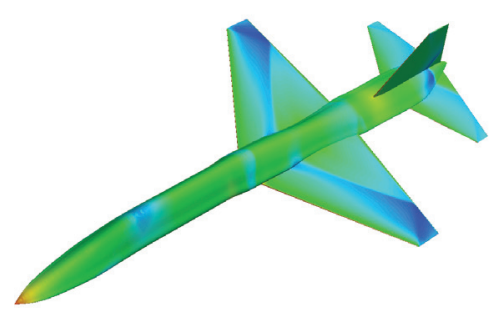

(c) RANS - no inlet

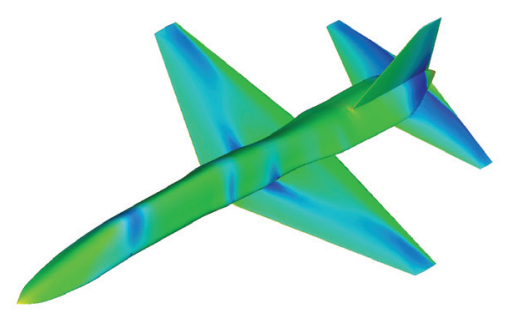

(d) Euler - no inlet

Figure 5. Drag coefficient versus Mach number. Flight test altitude is 30,000 ft. The surface pressure solutions correspond to $M=1$, and $\alpha=0$

Flight test drag for the T-38 clean configuration is available for Mach 0.2 to 1.4, at a test altitude of 30,000 feet. Note that, the vortex lattice method, Digital DATCOM, and Jet Designer are limited to predict the transonicspeed measurements. Euler and RANS predictions are compared with the experiments in Figure 5(a). The RANS simulations correspond to a grid with and without an air inlet geometry. Figure 5(a) shows that the Euler equations accurately predict the wave-drag trends, although the total drag values are underestimated due to the inviscid assumption. The T-38 fuselage has relatively large blunged sides for the two engine inlets. Figure 5(a) shows the RANS predictions of the grid with solid-wall type air inlets overpredicts flight test data, but the RANS simulations of the grid without the inlet geometry agrees well. Also, Figures 5(b)-(c) show the surface pressure comparisons for simulations at Mach 1.0. The shock waves originating from the fuselage/wing junction can be seen clearly in the figures.

The lift, drag, and pitching moment predictions of aerodynamic codes in Jet Designer and CEASIOM are also 
compared with wind tunnel and flight test measurements in Figure 6(a)-(c). Wind tunnel measurements are limited to ten degrees angle of attack, and only lift flight test data are available. The figures show the predicted lift from all aerodynamic codes agree well with the experiments at low angles of attack, but at the larger angles of attack only RANS code match well with the experimental lift. Also, results show that the vortex lattice and Jet designer lift are linear in angles of attack. More variation is seen in the predictions of drag and pitching moment. Euler predictions and the vortex lattice method underestimate the experimental drag due to the inviscid assumption, although the Euler drag increases at larger angles of attack as the code predicts the secondary boundary layer separation due to adverse pressure gradients. However, these predictions are not accurate, and hence the drag force is also inaccurate. Digital DATCOM and Jet Designer predictions have the effects of viscous corrections which are not reasonable at higher angles of attack. Figure 6(c) shows the Euler and RANS codes predict a more negative pitching moment slope with a positive pitching moment at zero degrees angle of attack. A possible reason is due to using a simplified model for the fuselage in the other codes. The pitching moment variation with angle of attack represent the pitch stability of the aircraft, and hence different flying qualities are expected from each aerodynamic code.

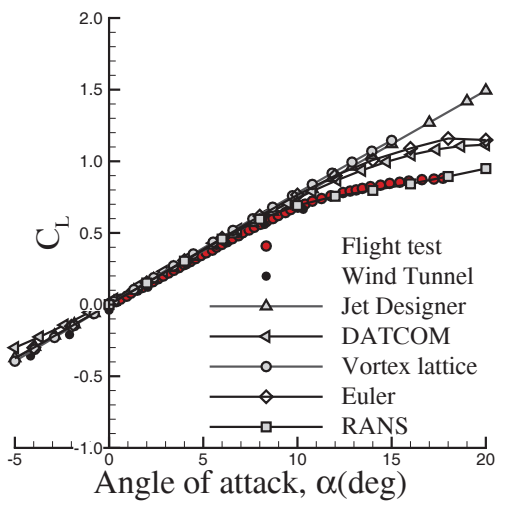

(a) lift coefficient

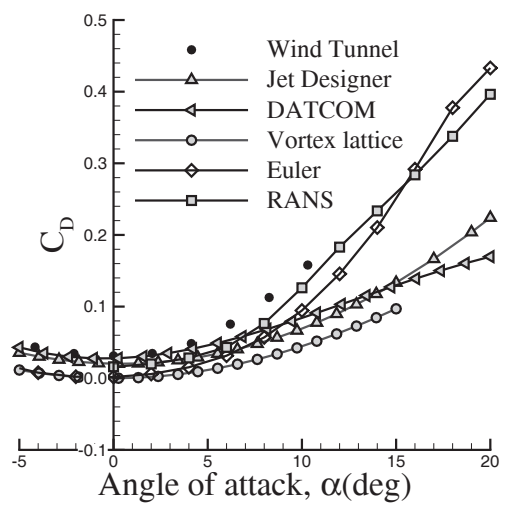

(b) drag coefficient

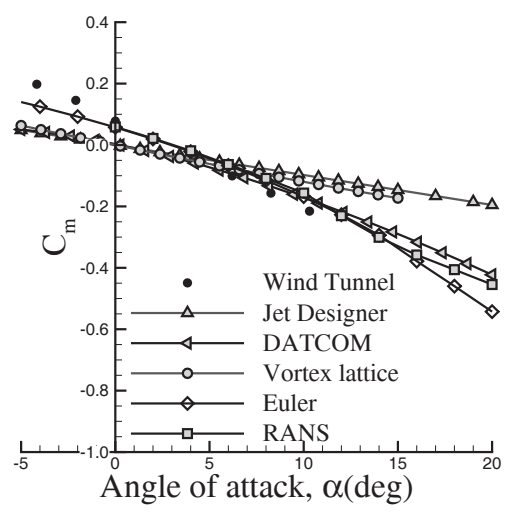

(c) pitching-moment coefficient

Figure 6. Validation of aero sources prediction at $M=0.4$

Flight test lift data are also available for a flapped T-38 aircraft at $M=0.4$ and 30,000 ft altitude with flap angles of $10^{\circ}$ and $45^{\circ}$. Figures 7 (a)-(b) compare the predictions of vortex lattice, Digital DATCOM, and the Euler code with these measurements. The figures show the lift is increased with increased flap angle, although the rate of increase falls at the larger flap angles. Note that the lift curve slope is nearly unchanged with flap deflection. DATCOM predict flap aerodynamics based on empirical relations which approximate aircraft aerodynamics from the vehicle geometry description and flight conditions. The figures show that DATCOM prediction match well with low and high flap angle measurements at low angles of attack. The vortex lattice code predicts a linear increment in lift due to flap angle changes, and hence the predictions at $45^{\circ}$ flap angle overpredicts the experiments. The way EDGE calculates the aerodynamics of control surface deflections is based on the use of transpiration boundary 
conditions to the clean configuration grid. A good match is found for low flap angles, but the approach is limited for prediction of large flap angles. For $10^{\circ}$ flap angle, and zero degrees angle of attack, the pressure solution of aircraft from vortex lattice and the Euler code are shown in Figure 8. Figures shows an increase in the negative pressure over the upper flap surface back of the hinge line of the T-38 flap.

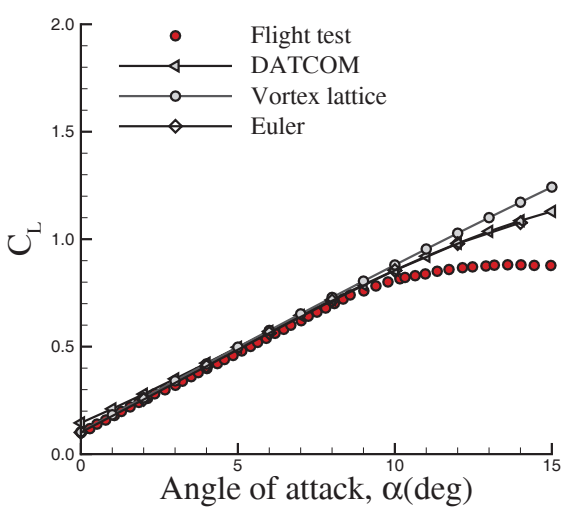

(a) flap angle $=10^{\circ}$

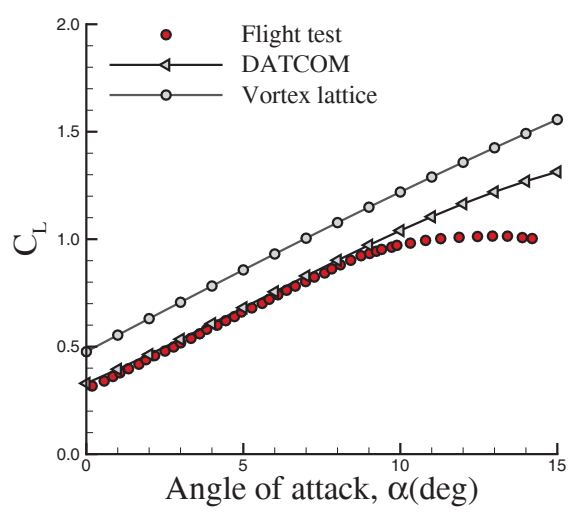

(b) flap angle $=45^{\circ}$

Figure 7. Flap deflections at $M=0.4, \alpha=0$ and $\beta=0$

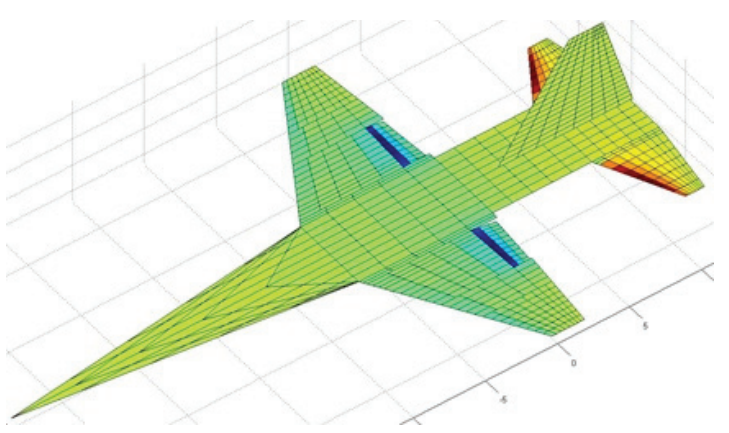

(a) Tornado

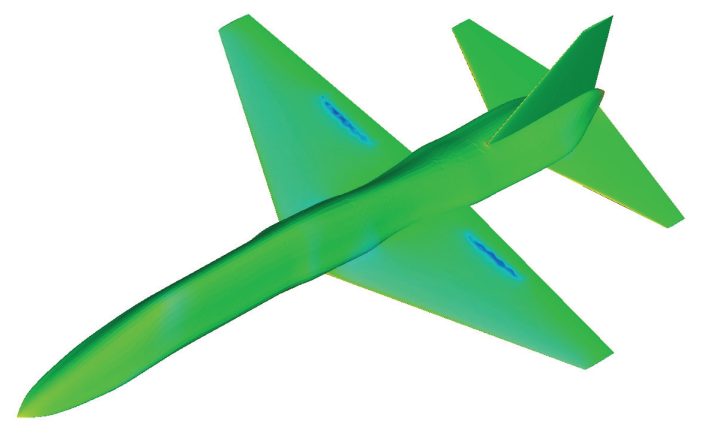

(b) Euler

Figure 8. Surface pressure solutions of 10 degrees flap deflection at $M=0.4, \alpha=0$

The Euler geometry is constructed from a small number of design parameters, and hence it would likely have rough joint and junction geometries. In this sense high fidelity simulations on low fidelity geometries may provide misleading information about the underlying properties of the design. This issue is investigated by comparing design prediction trends from the Euler code with available data. Figure 9 shows some examples: in Figure 9(a), the wing dihedral angle is varied from $-15^{\circ}$ to $15^{\circ}$, and then the side force derivative with respect to side slip angle from Jet Designer, DATCOM, and Euler are compared. The results show the general trends of the Euler code agree well with expected trends. Also, Figure 9(b) compares the design trends for wing sweep angle changes, and again the Euler code trend follows the trends of DATCOM and Jet Designer, although the absolute values are different. Finally, Figure 9(c) shows the effects of horizontal tail apex position on the pitching moment derivative with elevator deflection angle. This again confirms that application of the Euler code on the conceptual design follows the expected trends.

Aerodynamic tables took only a few seconds to generate using DATCOM and Jet Designer. It took only a few hours to generate these tables using the vortex lattice code. However, each Euler simulation would require a few hours which makes table generation a very expensive and time-consuming approach. A sampling method based 
on Kriging predicted mean squared error are used to reduced the computational cost. The table format is shown in Table. with angles of attack ranging from $0^{\circ}$ to $8^{\circ}$, Mach numbers of $0.1-0.8$, side-slip angle range of [-6 $-6^{\circ}$ ], and elevator, aileron, and rudder deflection angles of $-15^{\circ}$ and $15^{\circ}$. The sampling results of a table of angle-of-attack, Mach number and side-slip are shown in Figure 10; this table has 432 entries. The sampling starts from a $3 \times 3 \times 3$ factorial design, and then a Kriging model is created from the initial samples. Next, a sample is generated at the location where the maximum MSE occurs. The sampling stops as the total number of samples reaches 64. Figure 10(a) shows the root mean square error convergence at each iteration. At 64 iterations, the RMSE is sufficiently small. Also, Figure 10(b) shows the initial and updated angle of attack and Mach numbers. Figure 10(b) shows that the sampling approach tends to generate more samples at high speed regimes and large angles of attack.

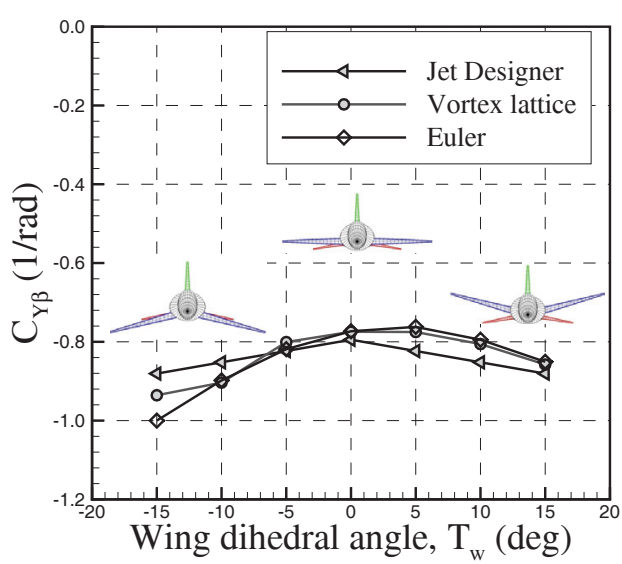

(a) Wing dihedral angle

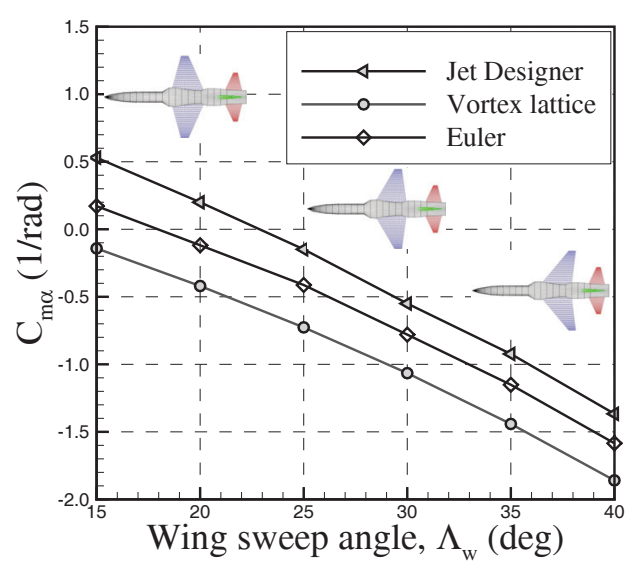

(b) Wing sweep angle

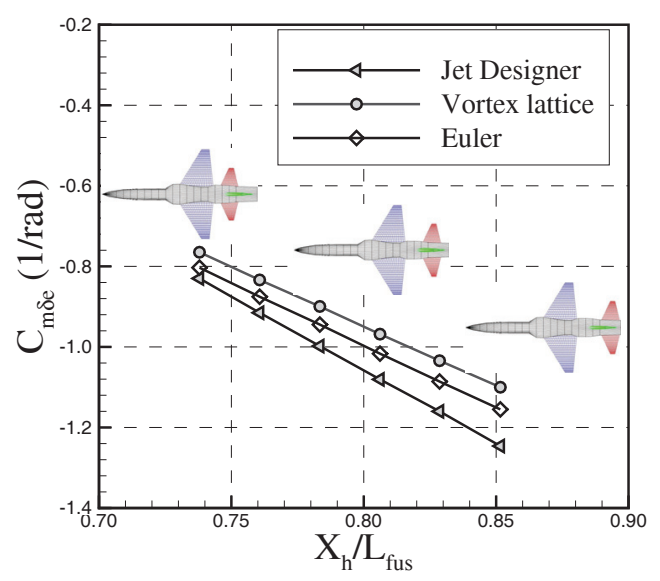

(c) Horizontal tail apex position

Figure 9. Prediction of geometry changes from different aero sources $M=0.3, \alpha=0$

As the aero tables were generated, they were then used to study the flying qualities of the T-38. Note that this analysis requires aircraft dynamic behavior. DATCOM, Jet Designer, and Tornado report dynamic derivatives data for the input geometry and flight conditions. For Euler, dynamic derivatives were calculated using Equations (9)(10). The Simulation and Dynamic Stability Analyzer (SDSA) code in CEASIOM was used to predict the flying qualities of aircraft. For this purpose, SDSA linearizes the rigid-aircraft equations of motion in matrix form around a trim point. The eigenvalues and eigenvectors of the Jacobian matrix are next computed in order to identify the typical modes of the aircraft responses and quantify their characteristics. Figure 11(a) shows the predicted Phugoid damping ratio of the T-38 aircraft using DATCOM, Tornado, and the Euler code for air speeds of $150-180 \mathrm{~m} / \mathrm{s}$ and an altitude of $25,000 \mathrm{ft}$. Also, for the aircraft with a test weight of $12,859 \mathrm{lb}$, and center of gravity at $19.4 \%$ 
MAC, the Phugoid damping ratio at this altitude and $155 \mathrm{~m} / \mathrm{s}$ was calculated and plotted in the figure. The results show all codes predict a damping ratio greater than 0.04 . Tornado predictions are nearly unchanged with air speed and overpredicts the flight test data. The Tornado code used in this paper has no compressibility effects. Also, DATCOM method predictions are limited for these high Mach numbers. Euler predictions provide a good match with flight test data. Figure 11(b) also shows the trim angle versus air speed at 25,000 ft. As the air speed increases, a smaller $\alpha$ is needed to trim the aircraft. The figure shows that the Euler tables predict a larger $\alpha$ compared with DATCOM and Tornado codes. DATCOM and Tornado are limited for these high speeds and they also predict a zero pitching moment at $\alpha=0$ possibly due to having a simplified fuselage model.

\section{Conclusions}

The results presented in this paper demonstrate the validity and feasibility of the simulation-based approach for aircraft conceptual design. The feasibility of this approach was achieved by undertaking these steps: 1) an automated solid modeling capability with a rapid mesh generator was used 2) aerodynamic tables were generated using a Kriging-based sampling approach with a reasonable cost 3) a transpiration boundary condition was used to change control surface deflection 4) and the CFD solutions of forced oscillations were used to calculate dynamic derivatives. The results show the limits of the designs based on spreadsheets to predict the high speed regime aerodynamics and fuselage effects into pitching moment. The simulation-based approach would allow the designers to develop a high fidelity aerodynamic model to evaluate stability and control of the aircraft prior to building the first prototype, and hence reducing the overall cost and limiting risks.

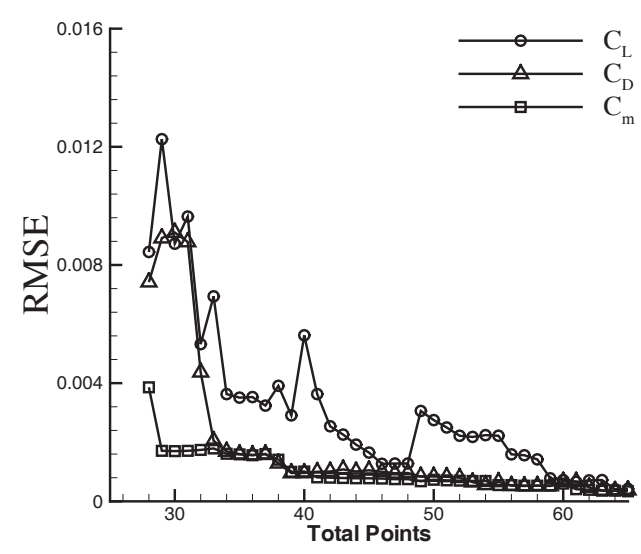

(a) RMSE convergence

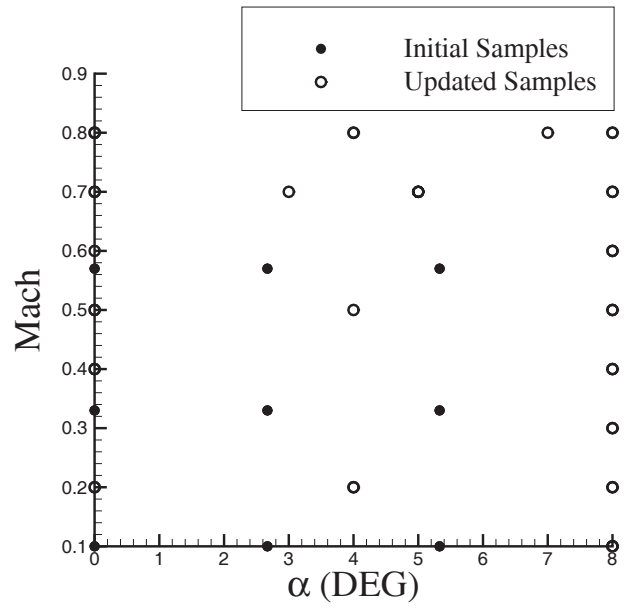

(b) Samples

Figure 10. RMSE convergence using $3^{3}+29$ sample points

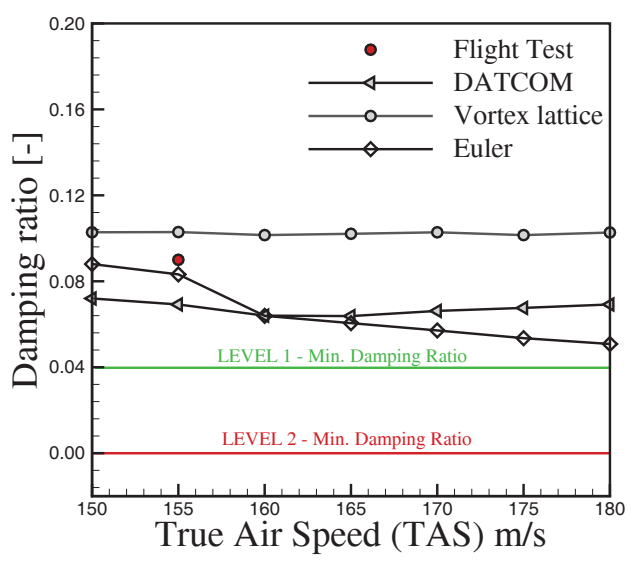

(a) Phugoid- Minimum damping ratio (MIL-F-8785C)

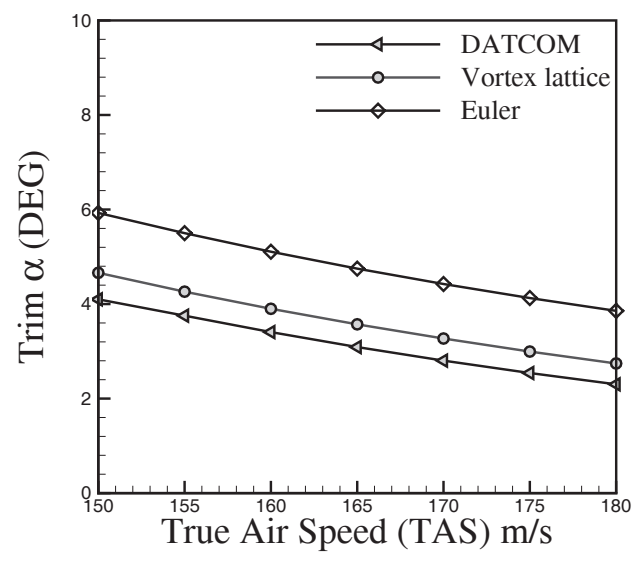

(b) Trim angle of attack

Figure 11. T-38 Phugoid damping ratio and trim angle of attack with speed- Altitude is $25,000 \mathrm{ft}$ 


\section{Acknowledgements}

Mehdi Ghoreyshi is supported by USAFA under contract FA70001320018. Their financial support is gratefully acknowledged. Acknowledgements are expressed to the Department of Defense High Performance Computing Modernization Program for providing computer time. The authors appreciate the support provided by the High Performance Computing Research Center at USAFA.

\section{References}

Alonso, J. J., Martins, J. R., Reuther, J. J., Haimes, R., \& Crawford, C. A. (2003, June). High-Fidelity Aerostructural Design Using a Parametric CAD-Based Model. AIAA Paper 2003 - 3429.

Austin, R. (2010). Unmanned Aircraft Systems: UAV Design, Development and Deployment. Chippenham, UK: Wiley.

Bowcutt, K. (2003, December). A Perspective on the Future of Aerospace Vehicle Design. AIAA Paper 2003 6957.

Bowers, A. H., Pahle, J. W., Wilson, R. J., Flick, B. C., \& Rood, R. L. (1996). An Overview of the NASA F- 18 High Alpha Research Vehicle. NASA TM-4772.

Berard, A., Rizzi, A., \& Isikveren, A. T. (2008, August). CADac: A New Geometry Construction Tool for Aerospace Vehicle Pre-Design and Conceptual Design. AIAA Paper 2008-6219.

Blake, W. B. (1985, October). Prediction of Fighter Aircraft Dynamic Derivatives Using Digital Datcom. AIAA Paper 85 - 4070.

Brandt, S. A., Stiles, R. J., Bertin, J. J., \& Whitford, R. (2004). Introduction to Aeronautics: A Design Perspective (2nd ed.). Reston, Virginia: AIAA Education Series.

Chambers, J. R., \& Hall, R. M. (2004). Historical Review of Uncommanded Lateral-Directional Motions at Transonic Conditions. Journal of Aircraft, 41(3), 436-447. http://dx.doi.org/10.2514/1.4470

Da Ronch, A., Ghoreyshi, M., Vallespin, D., Badcock, K. J., Mengmeng, Z., Oppelstrup, J., \& Rizzi, A. (2011, January). A Framework for Constrained Control Allocation Using CFD-based Tabular Data. AIAA Paper 2011-925.

Da Ronch, A., Vallespin, D., Ghoreyshi, M., \& Badcock, K. J. (2012). Evaluation of Dynamic Derivatives Using Computational Fluid Dynamics. AIAA Journal, 50(2), 470-484. http://dx.doi.org/10.2514/1.J051304

Eliasson, P. (2002). EDGE, a Navier-Stokes Solver for Unstructured Grids,. Finite Volumes for Complex Applications III: Problems and Perspectives. Hermes Penton Science, 527-534.

Ferreri, D. (2003). Marketing and Management in the High-Technology Sector: Strategies and Tactics in the Commercial Plane Industry. Westport, USA: Praeger Publishers.

Fielding, J. P. (1999). Introduction to Aircraft Design. Cambridge: Cambridge University Press.

Forsythe, J. R., Hoffmann, K. A., Cummings, R. M., \& Squires, K. D. (2002). Detached-Eddy Simulation with Compressibility Corrections Applied to a Supersonic Axisymmetric Base. Journal of Fluids Engineering, 124(4), 911-923. http://dx.doi.org/10.1115/1.1517572

Forsythe, J. R., Squires, K. D., Wurtzler, K. E., \& Spalart, P. R. (2004). Detached-Eddy Simulation of Fighter Aircraft at High Alpha. Journal of Aircraft, 41(2), 193-200. http://dx.doi.org/10.2514/6.2002-591

Forsythe, J. R., \& Woodson, S. H. (2005). Unsteady Computations of Abrupt Wing Stall Using Detached-Eddy Simulations. Journal of Aircraft, 42(3), 606-616. http://dx.doi.org/10.2514/1.2934

Ghoreyshi, M., Badcock, K. J., Da Ronch, A., Swift, A., Marques, S., \& Ames, N. (2011). Framework for Establishing the Limits of Tabular Aerodynamic Models for Flight Dynamics Analysis. Journal of Aircraft, 48(1), 42-55. http://dx.doi.org/10.2514/1.C001003

Ghoreyshi, M., Badcock, K. J., \& Woodgate, M. A. (2009). Accelerating the Numerical Generation of Aerodynamic Models for Flight Simulation. Journal of Aircraft, 46(3), 972-980. http://dx.doi.org/10.2514/1.39626

Ghoreyshi, M., Jirasek, A., \& Cummings, R. M. (2012). Computational Investigation into the Use of Response Functions for Aerodynamic-Load Modeling. AIAA Journal, 50(6), 1314-1327. 
http://dx.doi.org/10.2514/1.J051428

Gloudemans, J. R., Davis, P. C., \& Gelhausen, P. A. (1996, January). A Rapid Geometry Modeler For Conceptual Aircraft. AIAA Paper 1996 - 0052.

Gottlieb, J. J., \& Groth, C. P. T. (1988). Assessment of Riemann Solvers For Unsteady One-dimensional Inviscid Flows of Perfect Gasses. Journal of Computational Physics, 78(2), 437-458. http://dx.doi.org/10.1016/0021-9991(88)90059-9

Haimes. R. (1998). CAPRI: Computational Analysis Programming Interface. CAPRI technical guide, Massachusetts Institute of Technology.

Hall, R. M., Woodson, S. H., \& Chambers, J. R. (2005). Accomplishments of the Abrupt-Wing-Stall Program. Journal of Aircraft, 42(3), 653-660. http://dx.doi.org/10.2514/1.3631

Huenecke, K. (1987). Modern Combat Aircraft Design. Annapolis: Naval Institute Press.

Jameson, A. (1999). Re-Engineering the Design Process Through Computation. Journal of Aircraft, 36(1), 36-50. http://dx.doi.org/10.2514/2.2412

Jeans, T., McDaniel, D., Cummings, R. M., \& Mason, W. (2009). Aerodynamic Analysis of a Generic Fighter Using Delayed Detached-Eddy Simulations. Journal of Aircraft, 46(4), 1326-1339. http://dx.doi.org/10.2514/1.40955

Jirásek, A., \& Cummings, R. M. (2010, June-July). Reduced Order Modeling of X-31 Wind Tunnel Model Aerodynamic Loads. AIAA Paper 2010 - 4693.

Jones, D. R., Schonlau, M., \& Welch, W. J. (1998). Efficient Global Optimization of Expensive Black-Box Functions. Kluwer Academic Publications, 13(4), 455-492. http://dx.doi.org/10.1023/A:1008306431147

Klein, V., Murphy, P. C., Curry, T. J., \& Brandon, J. (1997, December). Analysis of Wind Tunnel Longitudinal Static and Oscillatory Data of the F-16XL Aircraft. NASA-TM-97-206276.

Kundu, A. (2010). Aircraft Design. New York, USA: Cambridge University Press.

Mackman, T. J., Allen, C. B., Ghoreyshi, M., \& Badcock, K. J. (2013). Comparison of Adaptive Sampling Methods for Generation of Surrogate Aerodynamic Models. AIAA Journal, 51(4), 797-808. http://dx.doi.org/10.2514/1.J051607

Marshall, C., Piper, D., Grillos, D., Green, K., Reichle, K., \& Brandt, S. A. (2005, September). Design, Analysis, and Subscale Testing of a Long-Range Supersonic Aircraft. AIAA Paper 2005 - 7137.

McCormick, D. J. (2002). An Analysis of using CFD in Conceptual Aircraft Design. Blacksburg, VA: M.S. Thesis, Dept. of Mechanical Engineering, Virginia Polytechnic Institute.

Melin, T. A. (2000, December). Vortex Lattice MATLAB Implementation for Linear Aerodynamic Wing Applications. MSc. Thesis, Royal Institute of Technology (KTH).

Morton, S. A., Forsythe, J. R., Mitchell, A. M., \& Hajek, D. (2002). Detached-Eddy Simulations and ReynoldsAveraged Navier-Stokes Simulations of Delta Wing Vortical Flowfields. Journal of Fluids Engineering, 124(4), 924-932. http://dx.doi.org/10.1115/1.1517570

Moses, R. W. (2000, April). Fin Buffeting Features of an Early F-22 Model. AIAA Paper 2000-1695.

Mueller, M. (1999). Thunderbird Milestones. Osceola: MBI Publising Company.

Murman, S. M., Chaderjian, N. M., \& Pandya, S. A. (2002, January). Generation of Aerodynamic Data Using Design of Experiment And Data Fusion Approach. AIAA Paper 2002-0259.

Nakahashi, K., Togashi, F., \& Sharov, D. (2000). Intergrid-Boundary Definition Method for Overset Unstructured Grid Approach. AIAA Journal, 38(11), 2077-2084. http://dx.doi.org/10.2514/2.869

Park, M. A., Green, L. L., Montgomery, R. C., \& Raney, D. L. (1999, June-July). Determination of Stability and Control Derivatives Using Computational Fluid Dynamics and Automatic Differentiation. AIAA Paper 99-316.

Potoczsky, A. S., \& Moses, R. W. (2005, April). An Analysis Method to Predict Tail Buffet Loads of Fighter Aircraft. AIAA Paper 2005-2291. 
Raymer, D. P. (2006). Aircraft Design: A Conceptual Approach (4th ed.). Reston, USA: AIAA Education Series.

Razgonyaev, V., \& Mason, W. H. (1995, January). An Evaluation of Aerodynamic Prediction Methods Applied to the XB-70 for Use in High Speed Aircraft Stability and Control System Design. AIAA Paper 95 - 0759.

Rodriguez, D. L., \& Sturdza, P. (2006, January). A Rapid Geometry Engine for Preliminary Aircraft Design. AIAA Paper 2006 - 929.

Rogers, S. E., Aftomis, M. J., Pandya, S. A., Chaderjian, N. M., Tejnil, E. T., \& Ahmad, J. U. (2003, June). Automated CFD Parameter Studies on Distributed Parallel Computers. AIAA paper 2003 - 4229.

Roskam, J. (2000). Airplane Design, Part 5: Preliminary Calculation of Aerodynamic, Thrust and Power Characteristics. Kansas: DARCorporation.

Strang, W. Z., Tomaro, R. F., \& Grismer, M. J. (1999, January). The Defining Methods of Cobalt: A Parallel, Implicit, Unstructured Euler/Navier-Stokes Flow Solver. AIAA Paper 1999 - 0786.

Tomac, M., \& Eller, D. (2010, September). From Geometry to CFD-Based Aerodynamic Derivatives-An Automated Approach. 27 ${ }^{\text {th }}$ International Congress of the Aeronautic Sciences, ICAS Paper 2010 - 2.4.3.

Tomaro, R. F., Strang, W. Z., \& Sankar, L. N (1997, January). An Implicit Algorithm For Solving Time Dependent Flows on Unstructured Grids. AIAA Paper 1997 - 0333.

Tyssel, L. (2000). The TRITET Grid Generation System. In Proceedings of the 10th International Conference on Numerical Grid Generation in Computational Field Simulations.

Vallespin, V., Da Ronch, A., Badcock, K. J., White, M., White, P., \& Ghoreyshi, M. (2012). Computational Fluid Dynamics Framework for Aerodynamic Model Assessment. Progress in Aerospace Sciences, 52(July), 2-18. http://dx.doi.org/10.1016/j.paerosci.2011.12.004

von Kaenel, R., Rizzi, A., Oppelstrup, J., Goetzendorf-Grabowski, T., Ghoreyshi, M., Cavagna, L., \& Berard, A. (2008, September). CEASIOM: Simulating Stability \& Control with CFD/CSM in Aircraft Conceptual Design. 26th International Congress of the Aeronautical Sciences, ICAS.

Wang, K. C., \& Iliff, K. W. (2004). Retrospective and Recent Examples of Aircraft Parameter Identification at NASA Dryden Flight Research Center. Journal of Aircraft, 41(4), 752-764. http://dx.doi.org/10.2514/1.332

Williams, J. E., \& Vukelich, S. R. (1979). The USAF Stability and Control Digital DATCOM, McDonnell Douglas Astronautics UTICS Company, AFFDL-TR-79-3032.

Yechout, T. R., Morris, L. A., Bossert, D. E., \& Hallgren, W. F. (2003) Introduction to Flight Mechanics: Performance, Static Stability, Dynamic Stability, and Classical Feedback Control. Reston, VA: AIAA Education Series.

\section{Copyrights}

This material is declared a work of the U.S. Government and is not subject to copyright protection in the United States, with first publication rights granted to the journal.

This is an open-access article distributed under the terms and conditions of the Creative Commons Attribution license (http://creativecommons.org/licenses/by/3.0/). 